\title{
Comparison of Corneal Wavefront-optimized and Wavefront-guided Alcohol-assisted Photorefractive Keratectomy Using Schwind Amaris 750S Laser for Myopia
}

\author{
Min-Ji Kang ${ }^{1}$, Jehyung Hwang ${ }^{1}$, So-Hyang Chung ${ }^{2}$ \\ ${ }^{I}$ Department of Ophthalmology, Sanggye Paik Hospital, Inje University College of Medicine, Seoul, Korea \\ ${ }^{2}$ Department of Ophthalmology and Visual Science, Seoul St. Mary's Hospital, The Catholic University of Korea College of Medicine,
} Seoul, Korea

Purpose: To compare the visual outcomes and corneal aberrations between wavefront-optimized (WFO) and corneal wavefront-guided (WFG) photorefractive keratectomy (PRK) in low to moderate myopia.

Methods: Twenty-seven eyes treated with WFO and 29 eyes treated with WFG PRK using a Schwind Amaris $750 S$ Excimer laser were included after 6 months of postoperative follow-up. Uncorrected distance visual acuity, corrected distance visual acuity, refractive errors, corneal higher-order aberrations (HOA) and corneal thickness obtained using a Scheimpflug system, and central ablation depth and volume were evaluated during the preoperative period and again at the postoperative 6-month visits.

Results: Postoperatively, uncorrected distance visual acuity, corrected distance visual acuity, manifest spherical equivalent, and refractive astigmatism were improved in both groups, and there was no statistically significant difference between the two groups. There was no significant difference in safety, efficacy, or predictability of the refractive outcome. Postoperative total corneal HOA root mean square (RMS), coma RMS, and spherical aberration were significantly increased in both groups. Among these, only spherical aberration showed a significant difference between the two groups, with greater increase in the WFO group at 6 months postoperatively. The changes in corneal HOA RMS and spherical aberration were smaller in the WFG group, and this benefit was marked in eyes with high HOA RMS $(\geq 0.4 \mu \mathrm{m})$ and spherical aberration $(\geq 0.2 \mu \mathrm{m})$. Even though ablation volume in the WFG group was much larger than that of the WFO group, there was no significant difference in postoperative central and peripheral corneal thickness between the two groups.

Conclusions: Both WFO and WFG PRK using a Schwind Amaris 750 S laser for low to moderate myopia were safe and effective at improving visual and refractive outcomes. However, WFG PRK induced fewer spherical aberrations than WFO PRK and may be more advantageous for eyes with high HOA root mean square or spherical aberration.

Key Words: Higher-order aberration, Spherical aberration, Wavefront-guided photorefractive keratectomy, Wavefront-optimized photorefractive keratectomy

Received: July 25, 2019 Final revision: October 21, 2019 Accepted: January 18, 2020

Corresponding Author: So-Hyang Chung, MD, PhD. Department of Ophthalmology, Seoul St. Mary's Hospital, The Catholic University of Korea College of Medicine, 222 Banpo-daero, Seocho-gu, Seoul 06591, Korea. Tel: 82-2-2258-1188, Fax: 82-2-599-7405, E-mail: chungsh@catholic.ac.kr 
Although myopic laser refractive surgery can improve uncorrected distance visual acuity (UDVA) by producing an oblate cornea and changing the focus of light that enters through the central cornea, visual quality is still often not optimal postoperatively. In such surgery, as the laser beam enters the periphery, some parts are reflected, and the circular beam becomes elliptical, which decreases the efficacy of the laser energy. All these factors can induce under-ablation of the peripheral cornea, resulting in increase of higher-order aberrations (HOA), especially spherical aberration [1-6].

To overcome this problem, wavefront-optimized (WFO) ablation was introduced to maintain an aspheric corneal shape by applying additional laser pulses to the peripheral cornea to reduce induction of spherical aberration [7]. Another procedure, wavefront-guided (WFG) ablation, was designed to reduce pre-existing HOA [8]. The Amaris Excimer laser system (Schwind eye-tech-solutions, Kleinostheim, Germany) provides three types of ablation profiles: 1) aberration-free to maintain an aspheric shape of the cornea in a similar manner as that used in WFO ablation, 2) corneal WFG ablation to reduce preoperative corneal HOA, and 3) ocular WFG ablation to reduce the degree of preoperative ocular HOA.

Previous studies about laser in situ keratomileusis (LASIK) have reported that spherical aberration and total corneal HOA did not decrease following Amaris WFO [912] but did decrease after WFG [13]. A recent study that applied transepithelial photorefractive keratectomy (PRK) in patients with myopia $<-10.00$ diopters (D) using an Amaris laser system reported that total corneal HOA increased in both WFO and WFG groups, but the amount of increase was smaller in the WFG patients. In addition, spherical aberration did not show any change postoperatively in either group [14].

In this study, we compared visual and refractive outcomes, corneal HOA, ablation volumes, and corneal thickness between WFO and corneal WFG alcohol-assisted PRK using an Amaris Excimer laser system in patients with low to moderate myopia.

\section{Materials and Methods}

\section{Patients}

The institutional review board at the Seoul St. Mary's
Hospital approved this retrospective comparative study (KC18RESI0240). Informed consents were obtained from all patients. The study followed the tenets of the Declaration of Helsinki. The patients enrolled in this study underwent WFO PRK (27 eyes in 15 patients) or corneal WFG PRK (29 eyes in 17 patients) targeting emmetropia using a Schwind Amaris 750S Excimer laser. The inclusion criteria were $<6.00 \mathrm{D}$ myopia, $<3.00 \mathrm{D}$ astigmatism, age between 20 and 40 years, stable refraction for at least 12 months, and corrected distance visual acuity (CDVA) of $20 / 20$ or better. We excluded eyes that had undergone previous ocular surgery; demonstrated potentially active, residual, or recurrent ocular disease; had corneal abnormalities; or had ever experienced trauma and also those in patients with other ocular medical conditions or who were taking systemic medications. All included patients were followed until at least 6 months postoperatively. Both eyes of each patient were enrolled, and the right-eye or left-eye data were randomly chosen in each group using randomization tables.

\section{Clinical parameters}

UDVA, CDVA, manifest refraction, corneal wavefront aberrations, and corneal thickness data were obtained preoperatively and again at 6 months postoperatively. Corneal wavefront aberrations at $6.0 \mathrm{~mm}$ pupil diameter, central corneal thickness (CCT), and peripheral corneal thickness (PCT) as mean values of superior, nasal, inferior, and temporal cornea in a $6.0-\mathrm{mm}$ area were evaluated using a Scheimpflug imaging system Pentacam (Oculus GmBH, Wetzlar, Germany). Root mean square (RMS) of total corneal HOA (3rd to 6 th order), coma $(3, \pm 1)$, trefoil $(3, \pm 3)$, and spherical aberrations $(4,0)$ were recorded. Coma $(3$, $\pm 1)$ and trefoil $(3, \pm 3)$ values were converted into RMS values to prevent the error of mirror symmetry, which is caused by different orientations of the eye [15].

\section{Surgical technique}

The surgery was performed by one surgeon (SHC) in Seoul St. Mary's Hospital. Each ablation profile was automatically designed using Optimized Refractive Keratectomy-Custom Ablation Manager software (Schwind eyetech-solutions) and a Schwind Amaris 750S Excimer laser. For the WFO group, the ablation profiles were based on aspheric aberration-neutral profiles to balance induction of 
spherical aberration. Centration was concentrated on the corneal vertex location obtained from the Keratron Scout topographer (Optikon, Rome, Italy). For the WFG group, all corneal HOA were treated with the corneal WFG ablation profiles obtained using a Keratron Scout topographer.

Preoperatively, $0.5 \%$ topical proparacaine hydrochloride (Alcaine; Alcon-Couvreur, Purrs, Belgium) anesthesia was instilled. The eyes were then scrubbed and draped, and a lid speculum was inserted. The epithelium was loosened and removed using $20 \%$ alcohol. Laser ablation was carried out on the exposed stroma; $0.02 \%$ mitomycin $\mathrm{C}$ was applied for 30 seconds, and the eyes were irrigated. A bandage contact lens (Acuvue Oasys; Johnson \& Johnson Vision Care, Jacksonville, FL, USA) was applied for four to five days until epithelial healing was complete. After surgery, 0.3\% topical gatifloxacin (Gatiflo; Senju Pharmaceuticals, Osaka, Japan) and 0.1\% fluorometholone (Ocumetholone; Samil Pharm., Seoul, Korea) were used four times a day for 1 month and then gradually tapered over the next 3 months.

\section{Statistical analysis}

Statistical analysis for normality was performed using the Kolmogorov-Smirnov test. Intragroup analysis to compare the preoperative and postoperative values was carried out using a paired $t$-test for normally distributed data and a Wilcoxon signed rank test for data with an abnormal distribution. Intergroup analysis was completed with an independent $t$-test for normally distributed data and a Mann-Whitney $U$-test for for data not normally distributed. Statistical analysis was performed using the IBM SPSS Statistics ver. 22.0 (IBM Corp., Armonk, NY, USA), and a $p$-value less than 0.05 was considered statistically significant.

\section{Results}

\section{Demographics}

There was no difference in age, sex, or orientation of eyes between the WFO and WFG groups. Preoperative UDVA, CDVA, manifest refraction sphere, manifest refraction cylinder, and manifest refraction spherical equivalent were also not significantly different. During the surgical procedure, optic zone size and total ablation zone size were not statistically different (Table 1). Corneal HOA

Table 1. Patient demographics features

\begin{tabular}{lccc}
\hline & Wavefront-optimized group & Corneal wavefront-guided group & $p$-value \\
\hline No. of eyes & 27 & 29 & \\
Age at operation $(\mathrm{yr})$ & $27.20 \pm 6.29(18$ to 41$)$ & $24.51 \pm 4.90(19$ to 36$)$ & 0.121 \\
Male : female & $15: 12(56: 44)$ & $16: 13(55: 45)$ & 0.909 \\
OD : OS & $14: 13(52: 48)$ & $1.43 \pm 0.63(0.20$ to 2.20$)$ & 0.817 \\
UDVA $(\operatorname{logMAR})$ & $1.34 \pm 0.56(0.50$ to 2.10$)$ & $-0.04 \pm 0.06(-0.10$ to 0.00$)$ & 0.986 \\
CDVA $(\operatorname{logMAR})$ & $-0.05 \pm 0.07(-0.10$ to 0.00$)$ & $-3.84 \pm 1.04(-5.00$ to -2.50$)$ & 0.998 \\
MR sphere $(\mathrm{D})$ & $-4.00 \pm 0.71(-5.00$ to -2.00$)$ & $-1.07 \pm 0.75(-2.75$ to 0.00$)$ & 0.496 \\
MR cylinder $(\mathrm{D})$ & $-1.02 \pm 0.78(-3.00$ to 0.00$)$ & $-4.35 \pm 0.89(-5.75$ to -3.25$)$ & 0.972 \\
MRSE $(\mathrm{D})$ & $-4.51 \pm 0.75(-5.75$ to -3.00$)$ & $663.0 \pm 34.70(603.5$ to 734.5$)$ & 0.890 \\
CCT $(\mu \mathrm{m})$ & $569.68 \pm 28.65(521$ to 617$)$ & $6.51 \pm 0.05(6.50$ to 6.70$)$ & 0.794 \\
PCT $(\mu \mathrm{m})$ & $663.22 \pm 31.55(617$ to 720.5$)$ & $7.85 \pm 0.25(7.55$ to 8.90$)$ & 0.116 \\
OZ $(\mathrm{mm})$ & $6.49 \pm 0.04(6.30$ to 6.50$)$ & & \\
TAZ $(\mathrm{mm})$ & $7.75 \pm 0.25(7.35$ to 8.40$)$ & &
\end{tabular}

Values are presented as number, mean \pm standard deviation (range), or number (\%).

$\mathrm{OD}=$ right eye; $\mathrm{OS}=$ left eye; UDVA = uncorrected distance visual acuity; logMAR = logarithm of the minimum angle of resolution; $\mathrm{CDVA}=$ corrected distance visual acuity; $\mathrm{MR}=$ manifest refraction; $\mathrm{D}=$ diopter; $\mathrm{MRSE}=$ manifest refraction spherical equivalent; $\mathrm{CCT}$ $=$ central corneal thickness; $\mathrm{PCT}=$ peripheral corneal thickness; $\mathrm{OZ}=$ optical zone; TAZ $=$ total ablation zone. 
Table 2. Preoperative and postoperative changes in corneal wavefront aberrations

\begin{tabular}{|c|c|c|c|c|}
\hline & & \multicolumn{3}{|c|}{ Preoperative } \\
\hline & & Wavefront-optimized group & Corneal wavefront-guided group & $p$-value \\
\hline \multirow{3}{*}{$\begin{array}{l}\text { Higher-order aberration RMS } \\
(\mu \mathrm{m})\end{array}$} & Baseline & $0.45 \pm 0.26(0.20$ to 0.70$)$ & $0.36 \pm 0.07(0.23$ to 0.59$)$ & 0.067 \\
\hline & 6 mon & $0.80 \pm 0.24(0.41$ to 1.41$)$ & $0.72 \pm 0.18(0.44$ to 1.09$)$ & 0.200 \\
\hline & $\Delta$ from baseline & $0.39 \pm 0.25(-0.12$ to 0.92$)$ & $0.35 \pm 0.19(0.07$ to 0.75$)$ & 0.559 \\
\hline \multirow{3}{*}{$\begin{array}{l}\text { Coma RMS } \\
(3, \pm 1)(\mu \mathrm{m})\end{array}$} & Baseline & $0.20 \pm 0.15(0.03$ to 0.37$)$ & $0.18 \pm 0.08(0.02$ to 0.35$)$ & 0.952 \\
\hline & 6 mon & $0.41 \pm 0.25(0.14$ to 1.15$)$ & $0.41 \pm 0.22(0.07$ to 0.91$)$ & 0.848 \\
\hline & $\Delta$ from baseline & $0.23 \pm 0.23(-0.03$ to 0.94$)$ & $0.22 \pm 0.25(-0.15$ to 0.72$)$ & 0.957 \\
\hline \multirow{3}{*}{$\begin{array}{l}\text { Trefoil RMS } \\
(3, \pm 3)(\mu \mathrm{m})\end{array}$} & Baseline & $0.21 \pm 0.19(0.01$ to 0.58$)$ & $0.15 \pm 0.07(0.02$ to 0.31$)$ & 0.344 \\
\hline & 6 mon & $0.22 \pm 0.11(0.03$ to 0.45$)$ & $0.20 \pm 0.13(0.05$ to 0.51$)$ & 0.759 \\
\hline & $\Delta$ from baseline & $0.03 \pm 0.15(-0.32$ to 0.39$)$ & $0.05 \pm 0.13(-0.12$ to 0.46$)$ & 0.656 \\
\hline \multirow{3}{*}{$\begin{array}{l}\text { Spherical aberration } \\
(4,0)(\mu \mathrm{m})\end{array}$} & Baseline & $0.20 \pm 0.07(-0.01$ to 0.34$)$ & $0.17 \pm 0.07(-0.01$ to 0.31$)$ & 0.120 \\
\hline & 6 mon & $0.51 \pm 0.15(0.29$ to 0.78$)$ & $0.41 \pm 0.12(0.16$ to 0.67$)$ & $0.011^{*}$ \\
\hline & $\Delta$ change from baseline & $0.30 \pm 0.15(0.05$ to 0.60$)$ & $0.19 \pm 0.14(-0.04$ to 0.68$)$ & $0.029^{*}$ \\
\hline
\end{tabular}

Values are presented as mean \pm standard deviation (range).

$\mathrm{RMS}=$ root mean square; $\Delta=$ difference (postoperative - preoperative).

*Statistical significance.

Table 3. Postoperative visual and refractive outcomes at 6 months

\begin{tabular}{lccc}
\hline & Wavefront-optimized group & Corneal wavefront-guided group & $p$-value \\
\hline UDVA (logMAR) & $0.02 \pm 0.07(-0.10$ to 0.20$)$ & $0.01 \pm 0.06(-0.10$ to 0.20$)$ & 0.549 \\
CDVA (logMAR) & $-0.06 \pm 0.02(-0.10$ to 0.10$)$ & $-0.05 \pm 0.02(-0.10$ to 0.10$)$ & 0.916 \\
MR sphere (D) & $0.03 \pm 0.66(-1.75$ to 1.25$)$ & $0.18 \pm 0.55(-1.25$ to 1.75$)$ & 0.432 \\
MR cylinder (D) & $-0.33 \pm 0.40(-1.50$ to 0.00$)$ & $-0.33 \pm 0.39(-1.50$ to 0.00$)$ & 0.949 \\
MRSE (D) & $-0.13 \pm 0.61(-2.00$ to 0.87$)$ & $-1.41 \pm 0.61(-2.20$ to -0.20$)$ & 0.301 \\
$\triangle$ UDVA (logMAR) & $-1.32 \pm 0.55(-2.10$ to -0.50$)$ & $-0.03 \pm 0.02(-0.10$ to 0.10$)$ & 0.467 \\
$\triangle$ CDVA (logMAR) & $-0.04 \pm 0.02(-0.10$ to 0.10$)$ & $4.03 \pm 1.03(1.75$ to 6.75$)$ & 0.916 \\
$\triangle$ MR sphere (D) & $4.03 \pm 1.07(2.25$ to 6.25$)$ & $0.68 \pm 0.74(-0.75$ to 2.50$)$ & 0.988 \\
$\triangle$ MR cylinder (D) & $0.69 \pm 0.79(-0.25$ to 2.75$)$ & $4.37 \pm 0.90(2.37$ to 6.37$)$ & 0.861 \\
$\triangle$ MRSE (D) & $4.37 \pm 1.01(2.50$ to 6.12$)$ & & 1.000 \\
\hline
\end{tabular}

Values are presented as mean \pm standard deviation (range).

UDVA = uncorrected distance visual acuity; $\log M A R=$ logarithm of the minimum angle of resolution; CDVA = corrected distance visual acuity; $\mathrm{MR}=$ manifest refraction; $\mathrm{D}=$ diopter; $\mathrm{MRSE}=$ manifest refraction spherical equivalent; $\Delta=$ difference (postoperative preoperative).

RMS, coma RMS, trefoil RMS, and spherical aberration also showed no statistical difference between the two groups (Table 2).

\section{Postoperative visual acuity and refractive outcome}

Postoperatively, UDVA improved with statistical signifi- cance in both groups (all $p$-values $<0.05$ ), and there was no difference in UDVA and CDVA between the two groups (Table 3). The efficacy index, which is defined as the ratio of postoperative logMAR UDVA (converted to a decimal) to preoperative logMAR CDVA (converted to a decimal) was $0.85 \pm 0.12$ in the WFO group and $0.89 \pm 0.13$ in the WFG group, with no statistical difference (Fig. 1A). The 
A

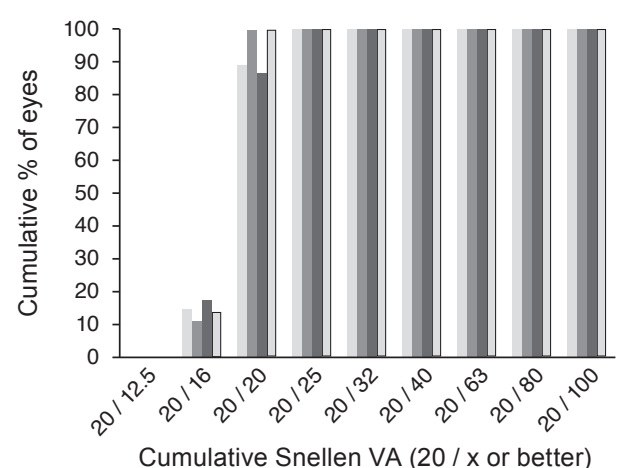

C

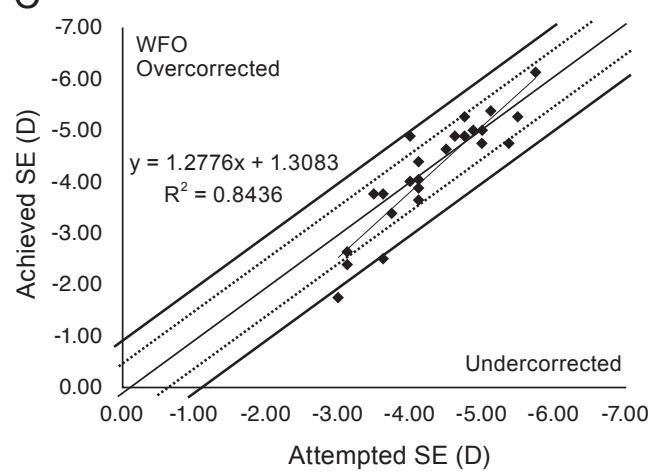

E

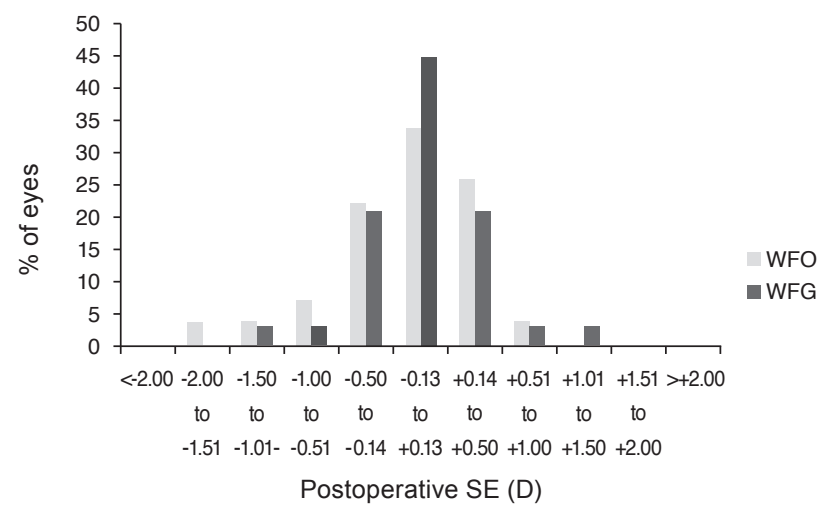

B

WFO postop UDVA

$\square$ WFO preop CDVA

-WFG postop UDVA $\square$ WFG preop CDVA

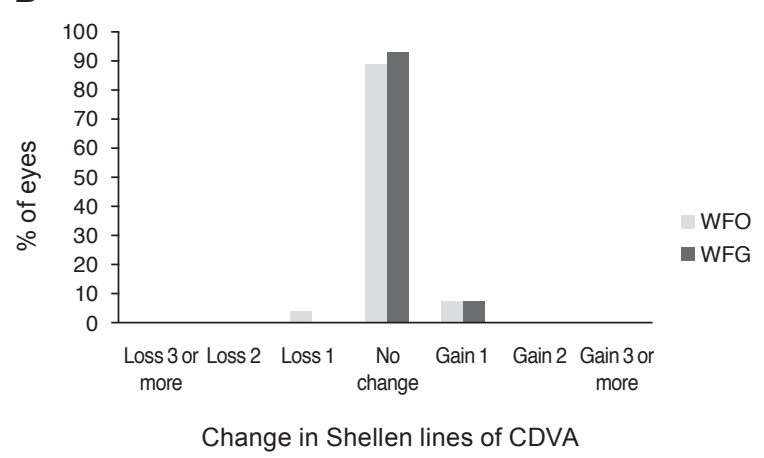

D

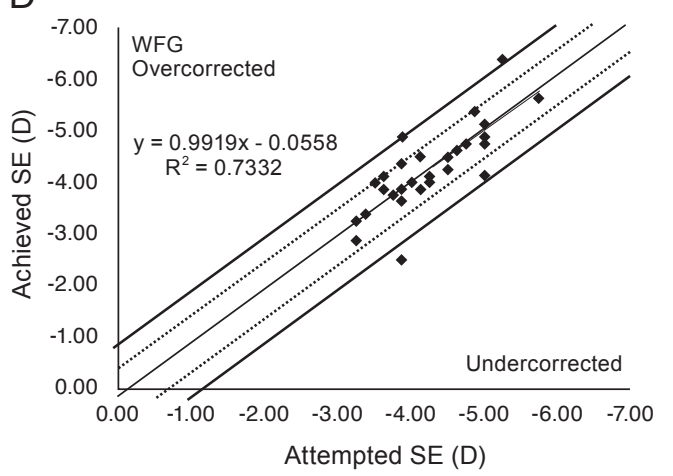

$\mathrm{F}$

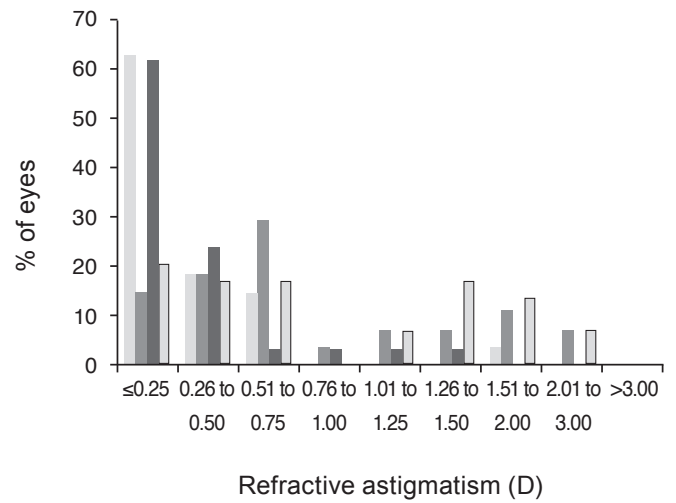

WFO postop WFO preop -WFG postop $\square$ WFG preop

Fig. 1. Visual and refractive outcomes. (A) Cumulative Snellen visual acuity (VA) of preoperative corrected distance visual acuity (CDVA) and 6-month postoperative uncorrected distance visual acuity (UDVA) in wavefront-optimized (WFO) and corneal wavefront-guided (WFG) groups. (B) Six-month postoperative changes of CDVA in Snellen lines. (C) Attempted versus achieved spherical equivalent (SE) in the WFO group. (D) Attempted versus achieved SE in the WFG group. (E) Accuracy of the 6-month postoperative SE in WFO and WFG groups. (F) Distribution of refractive cylinders in WFO and WFG groups. postop = postoperative; preop = preoperative; $\mathrm{D}=$ diopter.

percentage of CDVA that was the same or better than one Snellen line compared with the preoperative value was $96 \%$ in the WFO group and $100 \%$ in the WFG group. Four percent of WFO group patients and none of the WFG group patients experienced a one-Snellen-line loss in CDVA, and there were no cases with CDVA loss of more than two Snellen lines (Fig. 1B). The safety index, which is defined as the ratio of postoperative to preoperative CDVA, was $1.02 \pm 0.11$ in the WFO group and $1.02 \pm 0.13$ in the WFG group, and there was no significant difference between groups.

The linear regression graph of attempted versus achieved spherical equivalent (SE) revealed a slope of 1.2776 and a coefficient of determination $\left(\mathrm{R}^{2}\right)$ of 0.8436 in the WFO 


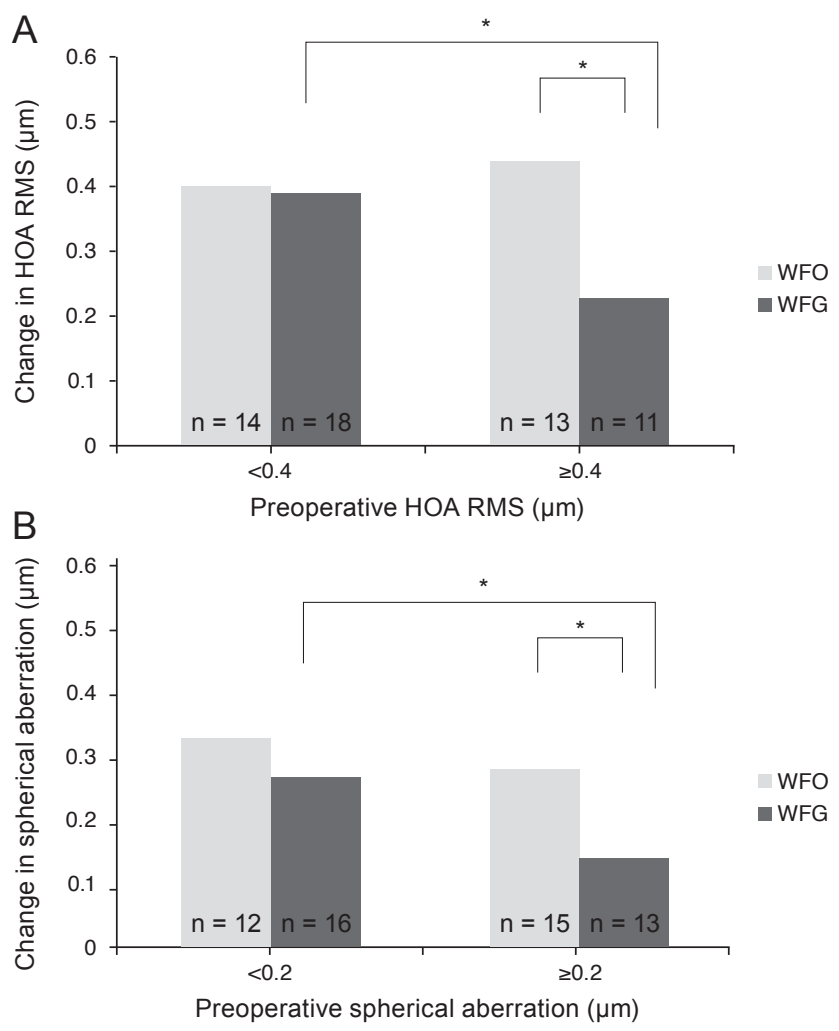

Fig. 2. Higher order aberration outcomes. (A) Histogram of change in total corneal higher order aberration (HOA) root mean square (RMS) according to preoperative value. (B) Histogram of change in spherical aberration according to preoperative value. $\mathrm{WFO}=$ wavefront-optimized; $\mathrm{WFG}=$ corneal wavefront-guided . ${ }^{*}$ Statistical significance.

group (Fig. 1C), while these same values were 0.9919 and 0.7332 in the WFG group, respectively (Fig. 1D). The proportion of SEs within $\pm 0.5 \mathrm{D}$ at 6 months postoperatively was $81 \%$ in the WFO group and $87 \%$ in the WFG group, and SEs within $\pm 1.0 \mathrm{D}$ were recorded in $92 \%$ and $93 \%$ of the WFO and WFG groups, respectively (Fig. 1E). Proportions of patients with refractive astigmatism within $\pm 0.5 \mathrm{D}$ were $82 \%$ and $86 \%$ in the WFO and WFG group patients, respectively (Fig. 1F). There was no difference in postoperative value or difference value (postoperative - preoperative) for UDVA, CDVA, sphere, cylinder, and SE between the two groups at 6 months postoperatively (Table 3).

\section{Postoperative corneal wavefront aberration}

Postoperative total corneal HOA RMS, coma RMS, and spherical aberration were significantly increased in both groups compared with the preoperative values (all $p$-values
$<0.001)$. Among these, only spherical aberration showed a significant difference between the two groups, with the WFO group demonstrating a greater increase at 6 months postoperatively $(0.51 \pm 0.15 \mu \mathrm{m}$ and $0.41 \pm 0.12 \mu \mathrm{m}$ in the WFO and WFG groups, respectively, $p=0.011$ ). The amount of change (postoperative - preoperative) was also significantly higher in the WFO group compared with the WFG group $(0.30 \pm 0.15 \mu \mathrm{m}$ and $0.19 \pm 0.14 \mu \mathrm{m}$, respectively: $p=0.029)$ (Table 2).

When the amount of change in total corneal HOA RMS was compared according to preoperative total RMS values, change in total RMS was smaller in the WFG group than in the WFO group for patients with $>0.4 \mu \mathrm{m}$ preoperative corneal HOA RMS ( $p=0.035$ ) (Fig. 2A). The change in spherical aberration was also smaller in the WFG group than in the WFO group in patients with $>0.2 \mu \mathrm{m}$ preoperative spherical aberration ( $p=0.035$ ) (Fig. 2B). In the WFG group, the observed changes in total HOA RMS and spherical aberration were smaller in patients with higher preoperative values than in those with lower values $(p<0.05)$ (Fig. 2A, 2B). These results indicate that WFG ablation induced lesser total HOA and spherical aberration in patients with higher preoperative values in myopic PRK.

\section{Ablation profile and corneal thickness}

There was no difference in central ablation depth between groups $(72.23 \pm 15.40$ and $78.78 \pm 17.85 \mu \mathrm{m}$ in WFO and WFG groups, respectively) (Table 4). Even though ablation volume in the WFG group was much larger than that in the WFO group $(1,575.74 \pm 462.73$ and $1,881.62 \pm$ $776.75 \mu \mathrm{L}$, respectively, $p=0.025$ ), there was no difference in postoperative CCT and PCT or degree of CCT and PCT changes (Table 4).

\section{Discussion}

Conventional myopic laser surgery can correct lower-order aberrations (defocus and astigmatism) and improve visual outcomes, although preexisting and surgically induced HOA may result in deterioration of visual quality. As almost $80 \%$ of $\mathrm{HOA}$ in the human eye is derived from the cornea, and the eye's internal structure compensates somewhat for the change of HOA [16], many ablation profiles have been developed to reduce corneal HOA. The Amaris 
Table 4. Ablation profile and corneal thickness

\begin{tabular}{lccc}
\hline & Wavefront-optimized group & Corneal wavefront-guided group & $p$-value \\
\hline Central ablation depth $(\mu \mathrm{m})$ & $72.23 \pm 15.40(45.08$ to 99.73$)$ & $78.78 \pm 17.85(35.38$ to 89.95$)$ & 0.196 \\
Ablation volume $(\mu \mathrm{L})$ & $1,575.74 \pm 462.73(836.1$ to 2,443$)$ & $1,881.62 \pm 776.75(570$ to 2,438$)$ & $0.025^{*}$ \\
CCT & $493.0 \pm 30.47(414$ to 561$)$ & $493.0 \pm 33.16(451$ to 584$)$ & 0.928 \\
PCT & $643.0 \pm 32.7(595.0$ to 713.5$)$ & $640.0 \pm 32.8(589.5$ to 728.3$)$ & 0.957 \\
$\triangle$ CCT & $76.68 \pm 21.99(41$ to 124$)$ & $78.15 \pm 16.22(51$ to 113$)$ & 0.883 \\
$\triangle$ PCT & $22.15 \pm 12.04(2.5$ to 50$)$ & $23.42 \pm 13.29(2.75$ to 59.75$)$ & 0.853 \\
\hline
\end{tabular}

Values are presented as mean \pm standard deviation (range).

$\mathrm{CCT}=$ central corneal thickness; PCT $=$ peripheral corneal thickness; $\Delta=$ difference (postoperative - preoperative).

${ }^{*}$ Statistical significance.

laser system provides WFO ablation (aberration-free), which maintains corneal aspheric shape to prevent induction of spherical aberrations and also provides WFG ablation, which reduces preexisting HOA.

The present study in patients with low to moderate myopia demonstrated excellent UDVA, CDVA, and refractive outcomes in both WFO PRK and WFG PRK groups without any difference 6 months postoperatively. In terms of corneal HOA, postoperative total HOA RMS, coma RMS, and spherical aberration were significantly increased in both groups compared with their preoperative values. This postoperative increase in total HOA RMS occurred regardless of preoperative total HOA RMS value. One previous study of WFO laser subepithelial keratomileusis also revealed increased total HOA RMS and spherical aberration data [17]. Another study that compared WFO and WFG transepithelial PRK in patients with low to high myopia identified higher total RMS, coma, and trefoil scores in the WFO group at 6 months postoperatively [14]. In our alcohol-assisted PRK study, the WFO group demonstrated greater increase in spherical aberration that resulted in higher spherical aberration compared with the WFG group at the 6-month postoperative visit $(0.51 \pm 0.15$ and $0.41 \pm$ $0.12 \mu \mathrm{m}$, respectively). Because it has been widely reported that spherical aberration is the most likely HOA to affect image quality $[18,19]$, corneal WFG ablation might be more advantageous to obtain a satisfactory visual performance after PRK.

With regard to surgical profiles, optic zone size, total ablation zone, and central ablation depth were not different between the two groups. However, ablation volume was larger in the WFG group than in the WFO group $(1,881.62 \pm$ 776.75 and 1,575.74 $\pm 462.73 \mu \mathrm{L}$, respectively), which indi- cated that the greater degree of peripheral ablation that occurs during WFG could contribute to a smaller increase of spherical aberration in the WFG group compared with the WFO group. Even though ablation volume was larger in the WFG group, postoperative corneal thickness showed no difference between the two groups for either CCT and PCT. A previous study comparing CCT between WFO and WFG patients after transepithelial PRK showed that postoperative CCT was not different between the two groups, although the study did not evaluate ablation volume or PCT [14]. This discordance between reported ablation volume and corneal thickness led us to formulate two hypotheses. First, we posit that a larger ablation volume does not exert a statistically significant influence on corneal thickness in patients with low to moderate myopia. We presume that CCT or PCT might be different between two groups in high-myopia patients with larger ablation volume. Second, corneal epithelial remodeling toward a more original corneal shape might occur during the first 6 months postoperatively. One study demonstrated that epithelial remodeling is different in the center and periphery after myopic transepithelial PRK. In their study, the epithelium became thicker in the periphery compared with the center at 6 months postoperatively, indicating that the corneal surface takes on a negative, meniscus-like lenticular shape. Their study implied that this epithelial remodeling could result in a larger than expected degree of spherical aberration [20]. Accurate measurement of corneal epithelial thickness obtained using an RTVue Fourier-domain OCT system (Optovue, Fremont, CA, USA) or an Artemis VHFDU (ArcScan, Morrison, CO, USA) system in PRK patients is expected to confirm our second hypothesis in future studies.

While WFO treatment cannot include preoperative 
HOA, WFG treatment does account for preexisting HOA and allows integration of a customized ablation plan aimed at reducing preexisting HOA. A comparative study of WFO and WFG LASIK reported that postoperative HOA RMS had a different outcome according to preoperative HOA RMS value [21]. If preoperative HOA RMS was $<0.3$ $\mu \mathrm{m}$, there was no difference between the two groups. However, when preoperative HOA RMS was $>0.3 \mu \mathrm{m}$, the WFG treatment had a greater advantage in reducing preexisting HOA RMS. This cutoff value of $0.3 \mu \mathrm{m}$ was based on several reports that concluded that the average HOA RMS value in a normal population was around $0.3 \mu \mathrm{m}$ $[15,22,23]$. In contrast, other researchers have insisted that HOA RMS differs according to ethnicity [24-26]. In our population, mean total corneal HOA RMS was $0.38 \pm 0.09$ $\mu \mathrm{m}$; therefore, we subdivided the group with a cutoff value of $0.4 \mu \mathrm{m}$. The WFO group showed no difference following intergroup and intragroup analyses. Interestingly, the WFG group demonstrated a smaller increase in HOA RMS upon intergroup and intragroup analyses when preoperative HOA RMS was $>0.4 \mu \mathrm{m}$. We also analyzed the change in spherical aberration using a cutoff value of 0.2 $\mu \mathrm{m}$ because the preoperative mean spherical aberration was $0.19 \pm 0.07 \mu \mathrm{m}$ in our population group. The WFG group also showed a smaller increase of spherical aberration upon intergroup and intragroup analyses when preoperative HOA RMS was $>0.2 \mu \mathrm{m}$. These results imply that the WFG profile has more merit in eyes with high HOA RMS or spherical aberration values.

In conclusion, both WFO and WFG PRK showed excellent visual acuity and refractive outcomes in low to moderate myopia. In patients with high preoperative HOA, WFG PRK was more advantageous for postoperative visual quality.

\section{Conflict of Interest}

No potential conflict of interest relevant to this article was reported.

\section{Acknowledgements}

This study was supported by the Basic Science Research Program through the National Research Foundation of Ko- rea (NRF) funded by the Ministry of Education (2016R1A6A1A03010528).

\section{References}

1. Cano D, Barbero S, Marcos S. Comparison of real and computer-simulated outcomes of LASIK refractive surgery. J Opt Soc Am A Opt Image Sci Vis 2004;21:926-36.

2. Yoon G, Macrae S, Williams DR, Cox IG. Causes of spherical aberration induced by laser refractive surgery. J Cataract Refract Surg 2005;31:127-35.

3. Oliver KM, Hemenger RP, Corbett MC, et al. Corneal optical aberrations induced by photorefractive keratectomy. $J$ Refract Surg 1997;13:246-54.

4. Miller JM, Anwaruddin R, Straub J, Schwiegerling J. Higher order aberrations in normal, dilated, intraocular lens, and laser in situ keratomileusis corneas. J Refract Surg 2002;18:S579-83.

5. Moreno-Barriuso E, Lloves JM, Marcos S, et al. Ocular aberrations before and after myopic corneal refractive surgery: LASIK-induced changes measured with laser ray tracing. Invest Ophthalmol Vis Sci 2001;42:1396-403.

6. Oshika T, Klyce SD, Applegate RA, et al. Comparison of corneal wavefront aberrations after photorefractive keratectomy and laser in situ keratomileusis. Am J Ophthalmol 1999;127:1-7.

7. Biebesheimer JB, Kang TS, Huang CY, et al. Development of an advanced nomogram for myopic astigmatic wavefront-guided laser in situ keratomileusis (LASIK). Ophthalmic Surg Lasers Imaging 2011;42:241-7.

8. Phusitphoykai N, Tungsiripat T, Siriboonkoom J, Vongthongsri A. Comparison of conventional versus wavefront-guided laser in situ keratomileusis in the same patient. J Refract Surg 2003;19(2 Suppl):S217-20.

9. Al-Zeraid FM, Osuagwu UL. Induced higher-order aberrations after laser in situ keratomileusis (LASIK) performed with wavefront-guided IntraLase femtosecond laser in moderate to high astigmatism. BMC Ophthalmol 2016;16:29.

10. Bohac M, Biscevic A, Koncarevic M, et al. Comparison of Wavelight Allegretto Eye-Q and Schwind Amaris 750S excimer laser in treatment of high astigmatism. Graefes Arch Clin Exp Ophthalmol 2014;252:1679-86.

11. Arbelaez MC, Vidal C, Arba-Mosquera S. Excimer laser correction of moderate to high astigmatism with a non-wavefront-guided aberration-free ablation profile: six- 
month results. J Cataract Refract Surg 2009;35:1789-98.

12. Arba-Mosquera S, Arbelaez MC. Three-month clinical outcomes with static and dynamic cyclotorsion correction using the SCHWIND AMARIS. Cornea 2011;30:951-7.

13. Arbelaez MC, Vidal C, Arba-Mosquera S. Clinical outcomes of corneal wavefront customized ablation strategies with SCHWIND CAM in LASIK treatments. Ophthalmic Physiol Opt 2009;29:549-56.

14. Jun I, Kang DS, Tan J, et al. Comparison of clinical outcomes between wavefront-optimized versus corneal wavefront-guided transepithelial photorefractive keratectomy for myopic astigmatism. J Cataract Refract Surg 2017;43: 174-82.

15. Wang L, Koch DD. Ocular higher-order aberrations in individuals screened for refractive surgery. $J$ Cataract Refract Surg 2003;29:1896-903.

16. Gatinel D, Adam PA, Chaabouni S, et al. Comparison of corneal and total ocular aberrations before and after myopic LASIK. J Refract Surg 2010;26:333-40.

17. Camellin M, Arba Mosquera S. Aspheric optical zones: the effective optical zone with the SCHWIND AMARIS. $J$ Refract Surg 2011;27:135-46.

18. Applegate RA, Sarver EJ, Khemsara V. Are all aberrations equal? J Refract Surg 2002;18:S556-62.

19. Applegate RA, Ballentine C, Gross H, et al. Visual acuity as a function of Zernike mode and level of root mean square error. Optom Vis Sci 2003;80:97-105.

20. Hou J, Wang Y, Lei Y, et al. Corneal epithelial remodeling and its effect on corneal asphericity after transepithelial photorefractive keratectomy for myopia. J Ophthalmol 2016;2016:8582362.

21. Stonecipher KG, Kezirian GM. Wavefront-optimized versus wavefront-guided LASIK for myopic astigmatism with the ALLEGRETTO WAVE: three-month results of a prospective FDA trial. $J$ Refract Surg 2008;24:S424-30.

22. Netto MV, Ambrosio R Jr, Shen TT, Wilson SE. Wavefront analysis in normal refractive surgery candidates. $J$ Refract Surg 2005;21:332-8.

23. Salmon TO, van de Pol C. Normal-eye Zernike coefficients and root-mean-square wavefront errors. $J$ Cataract Refract Surg 2006;32:2064-74.

24. Wei RH, Lim L, Chan WK, Tan DT. Higher order ocular aberrations in eyes with myopia in a Chinese population. $J$ Refract Surg 2006;22:695-702.

25. Nakano EM, Bains H, Nakano K, et al. Wavefront analysis in Asian-Brazilians. J Refract Surg 2006;22(9 Suppl):S1024-6.

26. Lim KL, Fam HB. Ethnic differences in higher-order aberrations: spherical aberration in the South East Asian Chinese eye. J Cataract Refract Surg 2009;35:2144-8. 